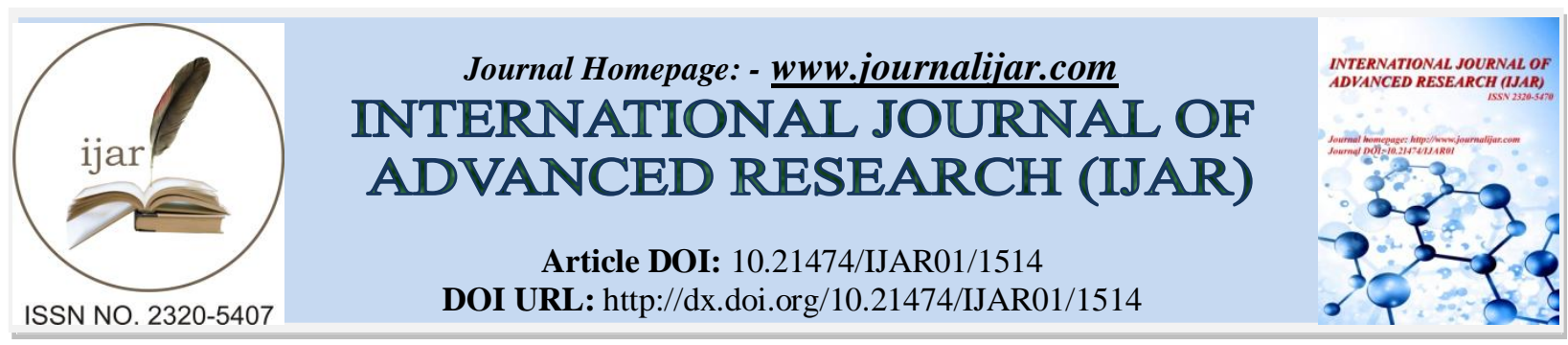

RESEARCH ARTICLE

\title{
PADDY STRAW COMPOSTING USING PHANEROCHAETE CHRYSOSPORIUM WITH VARIED TREATMENTS.
}

Navneet Kaur Gill ${ }^{*}$, Dr Sandeep Sharma ${ }^{2}$, Dr (Mrs) Maninder Arora ${ }^{3}$ and Dr (mrs) Surekha Bhatia ${ }^{4}$

1. Department of Microbiology, College of Basic Sciences and Humanities, Punjab Agricultural University, Ludhiana, Punjab, India.

2. Deparment of soil science, college of agriculture, Punjab,Agricultural University, Ludhiana-141004, Punjab, India.

3. Department of processing and food engineering, College of Agricultural Engineering and Technology, Punjab,Agricultural University, Ludhiana-141004, Punjab, India.

\section{Manuscript Info}

Manuscript History

Received: 16 July 2016

Final Accepted: 13 August 2016

Published: September 2016

Key words:-

Paddy straw, biodegradation,

Phanerochaete chrysosporium, composting

\section{Abstract}

Solid state fermentation of paddy straw was carried out with non-toxic fungi Phanerochaete chrysosporium (MTCC 787) with different treatments. Phanerochaete chrysosporium MTCC 787 was found to posses high endo- $\beta$-D-1, 4-glucanase $\left(0.561\right.$ and $\left.1.82 \mathrm{IUml}^{-1}\right)$, exo-1, 4 - $\beta$-glucanase $\left(0.917\right.$ and $\left.2.20 \mathrm{IUml}^{-1}\right)$ and xylanase $\left(0.917 \mathrm{Uml}^{-1}\right.$ and 2.20 $\left.\mathrm{Uml}^{-1}\right)$ activity, but low laccase $\left(0.245\right.$ and $\left.0.431 \mathrm{Uml}^{-1}\right)$ activity on $15^{\text {th }}$ and $30^{\text {th }}$ day of fermentation respectively. Phanerochaete chrysosporium (MTCC 787) was selected for paddy straw composting under field conditions with different treatments for 60 days. Treatment 5 (Paddy straw + Farm yard manure + Fungal culture (Phanerochaete chrysosporium MTCC 787) + Urea + Rock phosphate) was observed as the best treatment, as it favoured the efficient composting of paddy straw by degrading maximum hemicellulose (48\%), cellulose (39.2\%), lignin $(38 \%)$, silica content $(35 \%)$, total solids $(54 \%)$ and volatile solids (13.3\%). Scanning electron microscopy revealed that, untreated paddy straw exhibited a rigid and highly compact structure, whereas paddy straw treated with T5 (Paddy straw + Farmyard manure + Fungal culture + Urea + Rock phosphate) showed opening of the holo-cellulose fibrils due to creation of pores of different sizes.

Copy Right, IJAR, 2016,. All rights reserved.

\section{Introduction:-}

Paddy is India's major crop accounting for $20 \%$ of total world rice production. It's current production is 104.9 million tons (Annonymous, 2015). Annually a large amount of straw is accumulated as a by-product from rice cultivation, as straw makes up about $50 \%$ of the dry weight of the paddy. Paddy straw is one of the most abundant lignocellulosic waste on the earth. In India, total annual production of rice straw is estimated at 250 million tons (Anonymous, 2015). Farmers cannot incorporate paddy straw in the crop field because of its low degradation rate, disease infestation, unstable nutrients and reduced yield, caused by the short term negative effect of nitrogen immobilization (Pandey et al., 2009).They usually dispose of it through open field burning. As a result, carbon dioxide, carbon monoxide, methane, nitrous oxide and sulphur dioxide are emitted into the atmosphere, that can cause severe impact on human health (Gadde et al., 2009). The straw burning also destroys the soil texture. Paddy 
straw predominantly contains cellulose (35-40\%), hemicellulose (20-24\%), lignin (8-12\%), ash (14-16\%) and extractives (10-12\%).

Lignocellulosic materials are the most abundant agricultural residues in the world, primarily composed of cellulose (36.2\%), hemicelluloses (19.0\%) and lignin (9.9\%). Hydrolysis of these materials under natural condition is slow. Because lignin surrounds the cellulose and forms a physical barrier, which restricts microbial enzyme attack. Microorganisms including fungi as well as actinomycetes and other bacteria, have an important role in increasing digestibility of lignocellulolytic material. Biological treatment of agricultural residues is a new method for the improvement of their digestibility (Jalk et al., 1998). White-rot fungi, belonging to the wood-decaying basidiomycetes, as lignocellulolytic microorganisms are able to decompose and metabolize all plant cell constituents (cellulose, hemicellulose and lignin) by their enzymes (Eriksson et al., 1990). Most of the white-rot fungi degrade lignin and cellulose simultaneously. A selective white-rot fungus, Ceriporiopsis subvermispora is known to selectively degrade lignin in softwood and hardwood (Okano et al., 2005) and Phanerochaete chrysosporium, Pleurotus ostreatus, Ceriporiopsis subvermispora, Cyathus stercoreus can be used to increase the digestibility of paddy straw (Taniguchi et al., 2005).

Although cellulolytic fungi occur in all major fungal taxa (Coughlan, 1985), but there are relatively few groups of microorganisms that can produce the ligninolytic enzymes. The white-rot fungi enzyme complexe increases the accessibility of cell wall structure. Lignin is oxidised and degraded by a ligninase system (Rodrigues et al., 2008) composed by lignin peroxidase (LiP), manganese peroxidase (MnP) and laccase. In addition, cellulases, hemicellulases and esterases are also considered to be extremely important in the degradation process of lignocellulosic biomass (Panagiotou et al., 2007). These enzymes should act in synergy to facilitate the complete degradation of cell walls. Solid state fermentation is an advantageous method to degrade ligninocellulosic compounds and improve the digestibility. Fungi grown under these conditions not only bring better ligninolysis but also improve's its digestibility by enhancing the accessibility of holocellulose. Further, growth of fungal mycelium contributes in increasing the total protein content of the feed (Fazaeli, 2007).

Microbial composting is an effective environmentally sound alternative for degradation of paddy straw. All sorts of cereal straw may not be available for composting because of their fodder value. Paddy straw has limited use as animal feed because of its high oxalic acid and silica content. Composting of paddy straw enhance sustainable agriculture and environment protection by improving the physical, chemical and biological properties of soil (Mylavarapu and Zinati, 2009), which ultimately results in better plant growth and yield. The aim of composting is sanitation, eliminating pathogenic microorganisms and reducing the volume of the wastes (Zibiliske et al., 1998). Chemical and physicochemical pretreatment methods such as strong acid and steam explosion, respectively, break the lignin structure for enzymatic contact with the cellulose. However, such methods also bring about higher operational costs and hazardous waste (Sun and Cheng, 2002). The physical, chemical and physico-chemical treatments are still restricted in terms of safety concerns, costs and potential negative environmental consequences (Phutela et al., 2011). Hence biodegradation, serves as an attractive option that is both energy-saving and environmentally friendly (Scott et al., 1998). In this study, keeping in mind the poor nutritive quality of paddy straw, the experiments were designed to degrade lignin and cellulose by using lignocellulolytic fungi with different treatments, study the biochemical changes of straw constituents and improvement of its degradability.

\section{Material and methods:-}

\section{Enzyme Assay:-}

The activity of cellulases (FPU, CMCase and Xylanase) was estimated in the culture fifi ltrates after 15 and 30 days solid state fermentation of paddy straw method described by Sandhu and Kalra (1982). Laccase activity was assayed in the culture filtrates by the method of Dhaliwal et al (1991).

\section{Composting under field conditions:- Preparation of inoculums:-}

Two kg of sorghum (Sorghum bicolor) grains were soaked in water for three hours. After soaking, water was decanted out. The grains were boiled in 0.2 per cent dextrose solution for 15 minutes. Sorghum grains were transferred into conical flasks $(250 \mathrm{ml})$ at the rate of $70 \mathrm{~g}$ each. Addition of $\mathrm{CaSO}_{4}$ and $\mathrm{CaCO}_{3}$ was done at the rate of $2 \%$ and $4 \%$, respectively and autoclaved at $120^{\circ} \mathrm{C}$ at $15 \mathrm{lbs}$ psi for 15 minutes. Flasks containing grains were inoculated with $5 \mathrm{~mm}$ discs of 5 days old fungal cultures and mixed by hand shaking. Incubate flasks at $28 \pm 2^{\circ} \mathrm{C}$ for 7 days. 


\section{Preparation of compost:-}

Paddy straw for composting was collected from the experimental area of Department of Soil Science, PAU, Ludhiana.

Method:-

Unchopped paddy straw was weighed and filled in the pits of $29.5 \times 10 \mathrm{~cm}^{2}$ in length and breath. Pits were inoculated with the selected fungal culture at the rate of $5.0 \%(\mathrm{w} / \mathrm{w})$ and mixed throughly. Then the paddy straw was amended with FYM (3:1), urea (1\%), Rock phosphate (1\%) individually. The moisture content was maintained at $60 \%$ by adding water at different intervals of composting. The material was allowed to decompose for three months and turnings were given at 15 and 30 days of composting (Mishra et al 2013). Sampling was done after 0, 15, 30, 60 days to analyse the change in proximate and chemical composition of paddy straw (AOAC, 2000).

\section{Treatments for composting:-}

T0- Untreated Paddy straw (control)

T1- Paddy straw + FYM(3:1)

T2- Paddy straw + Fungal culture (5\%)

T3- Paddy straw + FYM(3:1) + Fungal culture (5\%)

T4- Paddy straw + FYM(3:1) + Fungal culture $(5 \%)+$ Urea $(1 \%)$

T5- Paddy straw + FYM(3:1) + Fungal culture (5\%) + Urea (1\%) + Rock phosphate (1\%)

\section{Scanning electron microscopy of paddy straw:-}

Paddy straw was immersed in $2.5 \%$ cacodylate buffered glutaraldehyde and kept at $4^{0} \mathrm{C}$ for 24 hours. The cells were washed with $0.1 \mathrm{M}$ cacodylate buffer three times for 15 minutes each at $4^{0} \mathrm{C}$. Secondary fixation was done with $1 \%$ osmium tetraoxide for 2 hours. Again three washings were done with $0.1 \mathrm{M}$ cacodylate buffer each for 15 minutes at $4^{0} \mathrm{C}$. After washing, dehydration was done three times with $30 \%, 50 \%, 70 \%, 80 \%$ and $90 \%$ ethanol each for 15 minutes and the dehydration step was completed by immersing samples in 100\% ethanol (three times) each for 15 minutes. Finally, ethanol was decanted and the sample was placed in a desiccator for drying. Then the sample was placed on stub and sputter coated with gold ion sputter coater. The samples were imaged in Hitachi S-3400N SEM at $15 \mathrm{kV}$ acceleration voltage.

\section{Statistical analysis:-}

Data was analysed statistically using Analysis of Variance (ANOVA) appropriate for factorial experiment in completely randomized design, further mean separation of treatment effects was accomplished by using Fisher's protected least significant difference test. All data analysis was carried out by using SAS-software.

\section{Results and Discussion:-}

Extracellular lignocellulolytic enzymatic assay:-

The activity of cellulase, xylanase and laccase was estimated during solid state fermentation of paddy straw using Phanerochaete chrysosporium MTCC 787 for 30 days of incubation at $28 \pm 2^{\circ} \mathrm{C}$. Cellulase is an enzyme complex comprising of chiefly endo- $\beta$-D-1, 4-glucanase (CMCase) and exo-1, 4- $\beta$-glucanase (FPU). Phanerochaete chrysosporium MTCC 787 was found to posses endo- $\beta$-D-1, 4-glucanase $\left(0.561\right.$ and $\left.1.82 \mathrm{IUml}^{-1}\right)$, exo-1, 4- $\beta$-glucanase $\left(0.917\right.$ and $\left.2.20 \mathrm{IUml}^{-1}\right)$ and xylanase $\left(0.917 \mathrm{Uml}^{-1}\right.$ and $\left.2.20 \mathrm{Uml}^{-1}\right)$ activity on $15^{\text {th }}$ and $30^{\text {th }}$ day of fermentation respectively. Laccase activity of Phanerochaete chrysosporium MTCC 787 on $15^{\text {th }}$ and $30^{\text {th }}$ day of fermentation was 0.245 and $0.431 \mathrm{Uml}^{-1}$ (Table 1). According to Chmelova et al (2011), lesser laccase activity was due to the toxicity of lignin degradation products, such as phenolic compounds, which probably reduced the growth of the organism and the production of laccase. However Mishra et al (2007) reported lesser CMCase (0.110 and $\left.0.429 \mathrm{IUml}^{-1}\right)$ and FPU $\left(0.403\right.$ and $\left.0.883 \mathrm{IUml}^{-1}\right)$ activity of $P$. chrysosporium after $7^{\text {th }}$ and $15^{\text {th }}$ day of incubation respectively. Prashanthi et al (2015) reported a xylananse activity of $6.52 \mathrm{IUml}^{-1}$ and $5.41 \mathrm{IUml}^{-1}$ by $P$. chrysosporium and Phlebia radiata respectively, where paddy straw was used as a substrate. Mishra et al (2007) reported a laccase activity of $0.131 \mathrm{IUml}^{-1}$ with paddy straw using $P$. chrysosporium NCIM 1093 on $15^{\text {th }}$ day of submerged fermentation.

\section{Effect of composting on proximate and chemical composition of paddy straw:-}

Phanerochaete chrysosporium (MTCC 787) was selected for paddy straw composting under field conditions with different treatments for 60 days and it's proximate and chemical composition was recorded on 15, 30,45 and 60 days of composting. Neutral detergent fibre (NDF) decreased in almost all treatments from $79.2 \%$ (control) to $45.6 \%$ after 60 
days of composting (Table.1). Similarly Acid Detergent Fibre (ADF) also decreased from 57.8\% (control) to 35.1\% after same days of composting. Maximum increase in percent degradation of NDF (41\%) and ADF (38.6\%) was observed in Treatment 5 (Paddy straw + FYM + Fungal culture + Urea + Rock phosphate) on $60^{\text {th }}$ day of composting. Maximum hemicellulose biodegradation was by T5 (38.6\%) closely followed by T4 (Paddy straw + FYM+ Fungal culture + Urea) (34.2\%), while minimum degradation was observed in T1 (Paddy straw + FYM), which was only $18.8 \%$ after 60 days of composting. A progressive decrease in cellulose content was observed in T5 $(39.2 \%)$ on $60^{\text {th }}$ day of composting as compared to control. Although, T4 showed maximum cellulose degradation on $45^{\text {th }}$ day of composting, but further composting led to increase in cellulose content. Maximum loss in lignin (38\%) was shown by Treatment 5 (Paddy straw + FYM + Fungal culture + Urea + Rock phosphate) followed by T4 (Paddy straw + FYM+ Fungal culture + Urea) (29.4\%) respectively after 60 days of composting. Goyal et al (2005) also recorded high activity of cellulase at $30^{\text {th }}$ day, while xylanase was found to be highest at $60^{\text {th }}$ day in composting mixture of sugarcane trash, cattle dung, pressmud and poultry droppings.

Lignin loss by T1 (Paddy straw + FYM) was only 6.5\%, within 60days of composting. In comparison to control, maximum degradation in silica was observed in T5 (35.5\%) followed by T4 $(29.5 \%)$ and T3 (Paddy straw + FYM + Fungal culture) (26\%) respectively after 60 days (Table.2). Beary et al (2002) have reported that a fungal bacterial consortium of Ceriporiopsis subvermispora and Cellulomonas sp enhances the sugarcane crop residue decomposition, when supplemented with $0.3 \%$ molasses. During 60 days of composting, all treatments showed decrease in total solids and volatile solids, but an increase in ash content was observed (Table. 3). Maximum change in total solids, ash content and volatile solids were brought by T5 (Paddy straw + FYM + Fungal culture + Urea + Rock phosphate) followed by T4 (Paddy straw + FYM+ Fungal culture + Urea) and T3 (Paddy straw + FYM + Fungal culture). Total solid contents gradually decreased from $29.6 \%$ (control) to $12.2 \%$, indicating a loss of $54 \%$ by T5 in 60 days. Similarly $45 \%$ and $42 \%$ of total solids were degraded by T4 and T3 respectively after same days of composting. Totally different trend was followed in case of ash content. In T5, ash content gradually increased from $16.2 \%$ to $27.1 \%$ after 60 days. Maximum degradation in Volatile solids was by T5 (13.3\%) on $60^{\text {th }}$ day of composting.

\section{Scanning electrom microscopy (SEM) of paddy straw:-}

Large fraction of holo-cellulose content was removed by composting, therefore, some physical changes were there in the straw. For this reason, SEM pictures of untreated paddy straw and paddy straw treated with T5 (Paddy straw + FYM + Fungal culture + Urea + Rock phosphate) were produced (Plate. 2). The distinct changes in surface structure were visible in the basic tissue of paddy straw. The untreated paddy straw exhibited a rigid and highly compact structure, whereas pretreated sample showed opening of the holo-cellulose fibrils due to creation of pores of different sizes. These structural analyses proved that composting of rice straw degraded the lignin and reduced the crystallinity of cellulose micofibrils. Micro-fibrils were separated from initial connected structure and are fully exposed, thus increasing the external surface area and porosity of paddy straw. Similar results were also reported by Yu et al (2009) reported that both morphological and structural characteristics were changed due to organic polar substances and inorganic silica partly dissolved, which leaves higher surface area with more pores of different sizes. 
Table 1:- Effect of different treatments on hemicellulose content of paddy straw during composting.

\begin{tabular}{|c|c|c|c|c|c|c|c|c|c|c|c|c|}
\hline \multirow{2}{*}{$\begin{array}{c}\text { Treatm } \\
\text { ents/ } \\
\text { days } \\
\text { after } \\
\text { incubat } \\
\text { ion } \\
\end{array}$} & \multicolumn{4}{|c|}{ NDF (\%) } & \multicolumn{4}{|c|}{ ADF (\%) } & \multicolumn{4}{|c|}{ Hemicellulose (\%) } \\
\hline & 15days & 30 days & 45 days & 60 days & 15 days & B0 days & 45 days & 60 days & 15 days & 30 days & 45 days & p0 days \\
\hline T0 & $\begin{array}{l}79.2 \pm \\
0.33\end{array}$ & $\begin{array}{c}78.1 \pm \\
0.34\end{array}$ & $\begin{array}{c}77.9 \pm \\
0.75\end{array}$ & $\begin{array}{c}77.4 \pm \\
0.65\end{array}$ & $\begin{array}{c}57.8 \pm \\
0.61\end{array}$ & $\begin{array}{c}57.9 \pm \\
0.45\end{array}$ & $\begin{array}{c}57.9 \pm \\
0.54\end{array}$ & $\begin{array}{c}57.2 \pm \\
0.85\end{array}$ & $\begin{array}{c}20.8 \pm \\
0.94\end{array}$ & $\begin{array}{c}20.6 \pm \\
0.43\end{array}$ & $\begin{array}{c}20.4 \pm \\
0.84\end{array}$ & $\begin{array}{c}20.2 \pm \\
0.33\end{array}$ \\
\hline T1 & $\begin{array}{c}77.3 \pm \\
0.45 \\
(1.13)\end{array}$ & $\begin{array}{c}71.4 \pm \\
0.37 \\
(8.57 \\
\downarrow)\end{array}$ & $\begin{array}{c}65.5 \pm \\
0.73 \\
(15.9 \\
\downarrow)\end{array}$ & $\begin{array}{c}69 \pm 0 . \\
94 \\
(13 \downarrow)\end{array}$ & $\begin{array}{c}55.9 \pm \\
0.34 \\
(3.4 \downarrow)\end{array}$ & $\begin{array}{c}52.2 \pm \\
0.23 \\
(9.8 \downarrow)\end{array}$ & $\begin{array}{c}52.2 \pm \\
0.32 \\
(9.8 \downarrow)\end{array}$ & $\begin{array}{c}52.6 \pm \\
0.23 \\
(11 \downarrow)\end{array}$ & $\begin{array}{c}22.4 \pm \\
1.34 \\
(7.6 \downarrow)\end{array}$ & $\begin{array}{l}19.2 \pm \\
0.07 \\
(7 \downarrow)\end{array}$ & $\begin{array}{c}17.5 \pm \\
0.92 \\
(10.1 \\
\downarrow)\end{array}$ & $\begin{array}{c}16.4 \pm \\
0.56 \\
(18.8 \\
\downarrow)\end{array}$ \\
\hline T2 & $\begin{array}{c}71.1 \pm \\
0.41 \\
(11.3 \\
\downarrow)\end{array}$ & $\begin{array}{c}62.5 \pm \\
0.42 \\
(20 \downarrow)\end{array}$ & $\begin{array}{c}58.3 \pm \\
0.33 \\
(25.1 \\
\downarrow)\end{array}$ & $\begin{array}{c}55.8 \pm \\
0.65 \\
(27.9 \\
\downarrow)\end{array}$ & $\begin{array}{c}53.1 \pm \\
1.02 \\
(8.1 \downarrow)\end{array}$ & $\begin{array}{c}47.4 \pm \\
0.65 \\
(18.1 \\
\downarrow)\end{array}$ & $\begin{array}{c}43.4 \pm \\
0.65 \\
(25 \downarrow)\end{array}$ & $\begin{array}{c}41.8 \pm \\
0.43 \\
(30.4 \\
\downarrow)\end{array}$ & $\begin{array}{c}18 \pm 0 . \\
84 \\
(13.4 \\
\downarrow)\end{array}$ & $\begin{array}{c}16.0 \pm \\
0.86 \\
(22.3 \\
\downarrow)\end{array}$ & $\begin{array}{c}14.9 \pm \\
0.65 \\
(26.9 \\
\downarrow)\end{array}$ & $\begin{array}{c}14 \pm 0 . \\
22 \\
(30.6 \\
\downarrow)\end{array}$ \\
\hline T3 & $\begin{array}{c}73.1 \pm \\
0.36 \\
(7.7 \downarrow)\end{array}$ & $\begin{array}{c}68.1 \pm \\
0.81 \\
(12.8 \\
\downarrow)\end{array}$ & $\begin{array}{c}60.1 \pm \\
1.41 \\
(27.5 \\
\downarrow)\end{array}$ & $\begin{array}{c}55.9 \pm \\
1.54 \\
(27.7 \\
\downarrow)\end{array}$ & $\begin{array}{c}54.6 \pm \\
0.43 \\
(5.53 \\
\downarrow)\end{array}$ & $\begin{array}{c}51.4 \pm \\
0.22 \\
(12.6 \\
\downarrow)\end{array}$ & $\begin{array}{c}43.1 \pm \\
0.73 \\
(27.6 \\
\downarrow)\end{array}$ & $\begin{array}{c}41.1 \pm \\
0.65 \\
(31.6 \\
\downarrow)\end{array}$ & $\begin{array}{c}18.5 \pm \\
2.45 \\
(7.21 \\
\downarrow)\end{array}$ & $\begin{array}{c}16.7 \pm \\
0.43 \\
(19 \downarrow)\end{array}$ & $\begin{array}{c}17 \pm 0 . \\
09 \\
(16.6 \\
\downarrow)\end{array}$ & $\begin{array}{c}14.8 \pm \\
0.83 \\
(32.7 \\
\downarrow)\end{array}$ \\
\hline T4 & $\begin{array}{c}68.9 \pm \\
0.81 \\
(13 \downarrow)\end{array}$ & $\begin{array}{c}61.1 \pm \\
0.18 \\
(21.8 \\
\downarrow)\end{array}$ & $\begin{array}{c}53.1 \pm \\
0.87 \\
(31.8 \\
\downarrow)\end{array}$ & $\begin{array}{c}51.0 \pm \\
0.54 \\
(34.4 \\
\downarrow)\end{array}$ & $\begin{array}{c}51.3 \pm \\
0.94 \\
(11.2 \\
\downarrow)\end{array}$ & $\begin{array}{c}45.6 \pm \\
0.23 \\
(21.2 \\
\downarrow)\end{array}$ & $\begin{array}{c}41 \pm 0 . \\
32 \\
(29.1 \\
\downarrow)\end{array}$ & $\begin{array}{c}38.6 \pm \\
0.39 \\
(36.5 \\
\downarrow)\end{array}$ & $\begin{array}{c}17.6 \pm \\
1.34 \\
(15.4 \\
\downarrow)\end{array}$ & $\begin{array}{c}15.3 \pm \\
0.08 \\
(25.7 \\
\downarrow)\end{array}$ & $\begin{array}{c}12.1 \pm \\
0.39 \\
(40.6 \\
\downarrow)\end{array}$ & $\begin{array}{c}12.4 \pm \\
0.32 \\
(38.6 \\
\downarrow)\end{array}$ \\
\hline T5 & $\begin{array}{c}69.8 \pm \\
0.09 \\
(11.8 \\
\downarrow)\end{array}$ & $\begin{array}{c}58 \pm 0 . \\
943 \\
(25 \downarrow)\end{array}$ & $\begin{array}{c}55.4 \pm \\
0.32 \\
(28.9 \\
\downarrow)\end{array}$ & $\begin{array}{c}45.6 \pm \\
1.67 \\
(41 \downarrow)\end{array}$ & $\begin{array}{c}51.3 \pm \\
0.44 \\
(11.2 \\
\downarrow)\end{array}$ & $\begin{array}{c}43.9 \pm \\
0.72 \\
(24.2 \\
\downarrow)\end{array}$ & $\begin{array}{c}41.6 \pm \\
0.76 \\
(28 \downarrow)\end{array}$ & $\begin{array}{c}35.1 \pm \\
1.56 \\
(38.6 \\
\downarrow)\end{array}$ & $\begin{array}{c}18.5 \pm \\
0.59 \\
(11 \downarrow)\end{array}$ & $\begin{array}{c}14.2 \pm \\
0.64 \\
(31 \downarrow)\end{array}$ & $\begin{array}{c}13.7 \pm \\
0.92 \\
(32.8 \\
\downarrow)\end{array}$ & $\begin{array}{c}10.5 \pm \\
0.68 \\
(48 \downarrow)\end{array}$ \\
\hline $\begin{array}{c}\mathrm{CD} @ 5 \\
\%\end{array}$ & 0.64 & 0.45 & 0.45 & 0.16 & 0.32 & 0.16 & 0.16 & 0.16 & 0.22 & 0.36 & 0.34 & 0.34 \\
\hline
\end{tabular}

T0- Untreated Paddy straw (control)

T1- Paddy straw + FYM(3:1)

T2- Paddy straw + Fungal culture $(5 \%)$

T3- Paddy straw + FYM $(3: 1)+$ Fungal culture $(5 \%)$

T4- Paddy straw + FYM(3:1) + Fungal culture $(5 \%)+$ Urea $(1 \%)$

T5- Paddy straw + FYM(3:1) + Fungal culture $(5 \%)+$ Urea $(1 \%)+$ Rock phosphate $(1 \%)$

CD: Critical difference for triplicate data

\pm values indicate $\%$ Standard error for triplicate data

Table 2:- Effect of different treatments on cellulose, lignin and silica content of paddy straw during composting.

\begin{tabular}{|c|c|c|c|c|c|c|c|c|c|c|c|c|}
\hline \multirow[b]{2}{*}{$\begin{array}{c}\text { Treatm } \\
\text { ents/ } \\
\text { Days } \\
\text { after } \\
\text { incubat } \\
\text { ion }\end{array}$} & \multicolumn{4}{|c|}{ Cellulose (\%) } & \multicolumn{4}{|c|}{$\operatorname{Lignin}(\%)$} & \multicolumn{4}{|c|}{ Silica(\%) } \\
\hline & $\begin{array}{c}\text { 15day } \\
\text { s }\end{array}$ & $\begin{array}{c}30 \\
\text { days }\end{array}$ & $\begin{array}{c}45 \\
\text { days }\end{array}$ & $\begin{array}{c}60 \\
\text { days }\end{array}$ & $\begin{array}{c}15 \\
\text { days }\end{array}$ & $\begin{array}{c}30 \\
\text { days }\end{array}$ & $\begin{array}{c}45 \\
\text { days }\end{array}$ & $\begin{array}{c}60 \\
\text { days }\end{array}$ & $\begin{array}{c}15 \\
\text { days }\end{array}$ & $\begin{array}{c}\text { 30 } \\
\text { days }\end{array}$ & $\begin{array}{c}45 \\
\text { days }\end{array}$ & $\begin{array}{c}60 \\
\text { days }\end{array}$ \\
\hline T0 & $\begin{array}{c}38.8 \pm \\
0.23\end{array}$ & $\begin{array}{c}38.3 \pm \\
0.09\end{array}$ & $\begin{array}{c}38.1 \pm \\
0.21\end{array}$ & $\begin{array}{c}38 \pm 0 \\
73\end{array}$ & $\begin{array}{c}7.9 \pm 0 \\
.13\end{array}$ & $\begin{array}{c}7.9 \pm 0 \\
.23\end{array}$ & $\begin{array}{c}7.8 \pm 0 \\
.07\end{array}$ & $\begin{array}{c}7.7 \pm 0 \\
.06\end{array}$ & $\begin{array}{c}12 \pm 0 \\
44\end{array}$ & $\begin{array}{c}12 \pm 0 \\
88\end{array}$ & $\begin{array}{c}11.8 \pm \\
0.07\end{array}$ & $\begin{array}{c}11.8 \pm \\
0.27\end{array}$ \\
\hline T1 & $\begin{array}{c}36.5 \pm \\
0.11 \\
(5.9 \downarrow)\end{array}$ & $\begin{array}{c}35.1 \pm \\
0.64 \\
(8.35 \downarrow \\
)\end{array}$ & $\begin{array}{c}35.4 \pm \\
0.43 \\
(7.08 \downarrow \\
)\end{array}$ & $\begin{array}{c}34.6 \pm \\
1.64 \\
(8.9 \downarrow)\end{array}$ & $\begin{array}{c}8.2 \pm 0 \\
.19 \\
(3.7 \uparrow \\
)\end{array}$ & $\begin{array}{c}7.3 \pm 0 \\
.44 \\
(7.6 \downarrow \\
)\end{array}$ & $\begin{array}{c}6.9 \pm 0 \\
.11 \\
(6.9 \downarrow \\
)\end{array}$ & $\begin{array}{c}7.8 \pm 0 \\
.11 \\
(6.5 \downarrow \\
)\end{array}$ & $\begin{array}{c}11.2 \pm \\
0.83 \\
(7.14 \downarrow \\
)\end{array}$ & $\begin{array}{c}10.6 \pm \\
0.54 \\
(11 \downarrow)\end{array}$ & $\begin{array}{c}10.1 \pm \\
0.74 \\
(11 \downarrow)\end{array}$ & $\begin{array}{c}10.2 \pm \\
0.35 \\
(13.5 \downarrow \\
)\end{array}$ \\
\hline T2 & $34.9 \pm$ & $33.3 \pm$ & $29.7 \pm$ & $26.8 \pm$ & $7.4 \pm 0$ & $6.3 \pm 0$ & $6.4 \pm 0$ & $5.9 \pm 0$ & $10.8 \pm$ & $9.5 \pm 0$. & $9.8 \pm 0$. & $9.1 \pm 0$. \\
\hline
\end{tabular}




\begin{tabular}{|c|c|c|c|c|c|c|c|c|c|c|c|c|}
\hline & 0.34 & 0.29 & 0.64 & 0.73 & .21 & .45 & .43 & .07 & 0.24 & 92 & 28 & 45 \\
& $(10.5 \downarrow$ & $(13 \downarrow)$ & $(22.04$ & $(29.4 \downarrow$ & $(6.38$ & $(20.5$ & $(17.9$ & $(23.3$ & $(10 \downarrow)$ & $(20.8 \downarrow$ & $(16.9 \downarrow$ & $(22.9 \downarrow$ \\
& ) & & $\downarrow)$ & ) & $\downarrow)$ & $\downarrow)$ & $\downarrow)$ & $\downarrow)$ & & ) & ) & ) \\
\hline T3 & $34.2 \pm$ & $35.6 \pm$ & $26.3 \pm$ & $26.3 \pm$ & $8 \pm 0.3$ & $6.1 \pm 0$ & $5.7 \pm 0$ & $6.1 \pm 0$ & $12.5 \pm$ & $9.7 \pm 0$. & $9.1 \pm 0$. & $8.74 \pm$ \\
& 0.56 & 0.93 & 1.76 & 0.4 & 2 & .18 & .76 & .34 & 0.85 & 08 & 72 & 082 \\
& $(12 \downarrow)$ & $(7.04 \downarrow$ & $(30.4 \downarrow$ & $(30.8 \downarrow$ & $(0.8 \uparrow$ & $(23 \downarrow)$ & $(26.9$ & $(20.7$ & $(4.2 \uparrow)$ & $(19.1 \downarrow$ & $(22.8 \downarrow$ & $(26 \downarrow)$ \\
& & ) & ) & ) & ) & & $\downarrow)$ & $\downarrow)$ & & ) & ) & \\
\hline T4 & $33.6 \pm$ & $30.2 \pm$ & $27.4 \pm$ & $25 \pm 0$. & $8.4 \pm 0$ & $6.5 \pm 0$ & $5 \pm 0.3$ & $5.3 \pm 0$ & $9.3 \pm 0$. & $9.2 \pm 0$. & $8.86 \pm$ & $8.32 \pm$ \\
& 0.38 & 0.18 & 0.36 & 23 & .68 & .69 & 2 & .63 & 3 & 63 & 0.34 & 0.24 \\
& $(13.4 \downarrow$ & $(21.1 \downarrow$ & $(28 \downarrow)$ & $(34.2 \downarrow$ & $(6.3 \uparrow$ & $(17.7$ & $(35.8$ & $(31.6$ & $(22.5 \downarrow$ & $(23 \downarrow)$ & $(24.9 \downarrow$ & $(29.5 \downarrow$ \\
& ) & ) & & ) & ) & $\downarrow)$ & $\downarrow)$ & $\downarrow)$ & ) & & ) & ) \\
\hline T5 & $32.3 \pm$ & $28.3 \pm$ & $28.5 \pm$ & $23.1 \pm$ & $8.7 \pm 0$ & $5.3 \pm 0$ & $5.0 \pm 0$ & $4.3 \pm 0$ & $10.3 \pm$ & $8.63 \pm$ & $8.32 \pm$ & $7.71 \pm$ \\
& 0.68 & 0.64 & 0.2 & 0.83 & .48 & .09 & .57 & .65 & 0.56 & 1.37 & 0.18 & 0.45 \\
& $(17 \downarrow)$ & $(26.1 \downarrow$ & $(25.7 \downarrow$ & $(39.2 \downarrow$ & $(9.2 \uparrow$ & $(33 \downarrow)$ & $(35.5$ & $(38 \downarrow)$ & $(14.1 \downarrow$ & $(28 \downarrow)$ & $(29.4 \downarrow$ & $(35 \downarrow)$ \\
& & ) & ) & ) & ) & & $\downarrow)$ & & ) & & ) & \\
\hline CD@5 & $\mathbf{0 . 1 6}$ & $\mathbf{0 . 3 2}$ & $\mathbf{0 . 3 2}$ & $\mathbf{0 . 3 2}$ & $\mathbf{0 . 3 4}$ & $\mathbf{0 . 3 5}$ & $\mathbf{0 . 2}$ & $\mathbf{0 . 3 5}$ & $\mathbf{0 . 3 4}$ & $\mathbf{0 . 3 5}$ & $\mathbf{0 . 3 2}$ & $\mathbf{0 . 3 4}$ \\
$\mathbf{\%}$ & & & & & & & & & & & & \\
\hline
\end{tabular}

T0- Untreated Paddy straw (control)

T1- Paddy straw + FYM(3:1)

T2- Paddy straw + Fungal culture (5\%)

T3- Paddy straw + FYM(3:1) + Fungal culture (5\%)

T4- Paddy straw + FYM $(3: 1)+$ Fungal culture $(5 \%)+$ Urea $(1 \%)$

T5- Paddy straw + FYM(3:1) + Fungal culture (5\%) + Urea (1\%) + Rock phosphate (1\%)

CD: Critical difference for triplicate data

Table 3:- Effect of different treatments on total solids, ash content and volatile solids of paddy straw during composting.

\begin{tabular}{|c|c|c|c|c|c|c|c|c|c|c|c|c|}
\hline \multirow[b]{2}{*}{$\begin{array}{c}\text { Treatmen } \\
\text { ts/days } \\
\text { after } \\
\text { incubatio } \\
n\end{array}$} & \multicolumn{4}{|c|}{ Total solids (\%) } & \multicolumn{4}{|c|}{$\operatorname{Ash}(\%)$} & \multicolumn{4}{|c|}{ volatile solids $(\%)$} \\
\hline & $\begin{array}{c}\text { 15da } \\
\text { ys }\end{array}$ & $\begin{array}{c}30 \\
\text { days }\end{array}$ & $\begin{array}{c}45 \\
\text { days }\end{array}$ & $\begin{array}{c}60 \\
\text { days }\end{array}$ & $\begin{array}{c}15 \\
\text { days }\end{array}$ & $\begin{array}{c}30 \\
\text { days }\end{array}$ & $\begin{array}{c}45 \\
\text { days }\end{array}$ & $\begin{array}{c}60 \\
\text { days }\end{array}$ & $\begin{array}{c}15 \\
\text { days }\end{array}$ & $\begin{array}{c}\text { 30 } \\
\text { days }\end{array}$ & $\begin{array}{c}45 \\
\text { days }\end{array}$ & $\begin{array}{c}60 \\
\text { days }\end{array}$ \\
\hline T0 & $\begin{array}{c}26.9 \pm \\
0.43\end{array}$ & $\begin{array}{c}26.7 \pm \\
0.28\end{array}$ & $\begin{array}{c}26.5 \pm \\
0.18\end{array}$ & $\begin{array}{c}26.4 \pm \\
0.51\end{array}$ & $\begin{array}{c}16.5 \pm \\
0.32\end{array}$ & $\begin{array}{c}16.3 \pm \\
0.12\end{array}$ & $\begin{array}{c}16.2 \pm \\
0.21\end{array}$ & $\begin{array}{c}15.9 \pm \\
0.12\end{array}$ & $\begin{array}{c}83.5 \pm \\
0.09\end{array}$ & $\begin{array}{c}83.7 \pm \\
0.12\end{array}$ & $\begin{array}{c}83.8 \pm \\
0.08\end{array}$ & $\begin{array}{c}84.1 \pm \\
0.15\end{array}$ \\
\hline T1 & $\begin{array}{c}25.3 \pm \\
1.32 \\
(5.9 \downarrow)\end{array}$ & $\begin{array}{c}24.6 \pm \\
0.65 \\
(7.86 \\
\downarrow)\end{array}$ & $\begin{array}{c}22.8 \pm \\
0.46 \\
(12 \downarrow)\end{array}$ & $\begin{array}{c}22.4 \pm \\
1.63 \\
(15.1 \\
\downarrow)\end{array}$ & $\begin{array}{c}18.3 \pm \\
0.04 \\
(10.9 \\
\downarrow)\end{array}$ & $\begin{array}{c}19.8 \pm \\
0.41 \\
(19.8 \\
\downarrow)\end{array}$ & $\begin{array}{c}19.3 \pm \\
0.53 \\
(26.4 \\
\downarrow)\end{array}$ & $\begin{array}{c}20.1 \pm \\
0.35 \\
(19.3 \\
\downarrow)\end{array}$ & $\begin{array}{c}81.7 \pm \\
0.14 \\
(2.1 \downarrow)\end{array}$ & $\begin{array}{c}81.2 \pm \\
0.06 \\
(3 \downarrow)\end{array}$ & $\begin{array}{c}80.7 \pm \\
0.13 \\
(3.69 \\
\downarrow)\end{array}$ & $\begin{array}{c}79.9 \pm \\
0.17 \\
(5 \downarrow)\end{array}$ \\
\hline T2 & $\begin{array}{c}24.8 \pm \\
0.45 \\
(7.8 \downarrow)\end{array}$ & $\begin{array}{c}20.3 \pm \\
0.18 \\
(24 \downarrow)\end{array}$ & $\begin{array}{c}17.3 \pm \\
0.63 \\
(34.6 \\
\downarrow)\end{array}$ & $\begin{array}{c}15.8 \pm \\
0.43 \\
(36 \downarrow)\end{array}$ & $\begin{array}{c}20.4 \pm \\
0.08 \\
(23.6 \\
\downarrow)\end{array}$ & $\begin{array}{c}24 \pm 0 . \\
85 \\
(48.4 \\
\downarrow)\end{array}$ & $\begin{array}{c}25.1 \pm \\
0.31 \\
(57.7 \\
\downarrow)\end{array}$ & $\begin{array}{c}25.3 \pm \\
0.62 \\
(59.1 \\
\downarrow)\end{array}$ & $\begin{array}{c}79.6 \pm \\
0.08 \\
(4.7 \downarrow)\end{array}$ & $\begin{array}{c}76 \pm 0 . \\
04 \\
(9.4 \downarrow)\end{array}$ & $\begin{array}{c}75.9 \pm \\
0.08 \\
(10.6 \\
\downarrow)\end{array}$ & $\begin{array}{c}74.7 \pm \\
0.13 \\
(11.1 \\
\downarrow)\end{array}$ \\
\hline T3 & $\begin{array}{c}23.6 \pm \\
0.14 \\
(15 \downarrow)\end{array}$ & $\begin{array}{c}19.2 \pm \\
0.91 \\
(28 \downarrow)\end{array}$ & $\begin{array}{c}16.5 \pm \\
0.54 \\
(37.7 \\
\downarrow)\end{array}$ & $\begin{array}{c}15.3 \pm \\
0.14 \\
(42 \downarrow)\end{array}$ & $\begin{array}{c}20.8 \pm \\
0.52 \\
(26 \downarrow)\end{array}$ & $\begin{array}{c}24.8 \pm \\
0.05 \\
(50.1 \\
\downarrow)\end{array}$ & $\begin{array}{c}28.3 \pm \\
0.82 \\
(78 \downarrow)\end{array}$ & $\begin{array}{c}26 \pm 0 . \\
26 \\
(63.5 \\
\downarrow)\end{array}$ & $\begin{array}{c}79.2 \pm \\
0.17 \\
(5.1 \downarrow)\end{array}$ & $\begin{array}{c}75.2 \pm \\
0.1 \\
(10.1 \\
\downarrow) \\
\end{array}$ & $\begin{array}{c}73.7 \pm \\
0.14 \\
(16 \downarrow)\end{array}$ & $\begin{array}{c}74 \pm 0 . \\
09 \\
(12 \downarrow)\end{array}$ \\
\hline T4 & $\begin{array}{c}22.9 \pm \\
0.19 \\
(12 \downarrow)\end{array}$ & $\begin{array}{c}18.4 \pm \\
0.54 \\
(31.1 \\
\downarrow)\end{array}$ & $\begin{array}{c}16.8 \pm \\
0.19 \\
(36.6 \\
\downarrow)\end{array}$ & $\begin{array}{c}14.6 \pm \\
0.82 \\
(45 \downarrow)\end{array}$ & $\begin{array}{c}21 \pm 0 . \\
42 \\
(27.2 \\
\downarrow)\end{array}$ & $\begin{array}{c}26 \pm 0 . \\
32 \\
(59.5 \\
\downarrow)\end{array}$ & $\begin{array}{c}24.8 \pm \\
1.64 \\
(53 \downarrow)\end{array}$ & $\begin{array}{c}25.6 \pm \\
0.16 \\
(61 \downarrow)\end{array}$ & $\begin{array}{c}79 \pm 0 . \\
17 \\
(5.4 \downarrow)\end{array}$ & $\begin{array}{c}73 \pm 0 . \\
18 \\
(12.7 \\
\downarrow)\end{array}$ & $\begin{array}{c}75.2 \pm \\
0.18 \\
(10.2 \\
\downarrow)\end{array}$ & $\begin{array}{c}74.4 \pm \\
0.11 \\
(11.5 \\
\downarrow)\end{array}$ \\
\hline T5 & $\begin{array}{c}23.6 \pm \\
0.05 \\
(12 \downarrow)\end{array}$ & $\begin{array}{c}17 \pm 0 . \\
8 \\
(36 \downarrow)\end{array}$ & $\begin{array}{c}14.8 \pm \\
0.53 \\
(44.2 \\
\downarrow) \\
\end{array}$ & $\begin{array}{c}12.2 \pm \\
0.43 \\
(54 \downarrow)\end{array}$ & $\begin{array}{c}22.5 \pm \\
0.25 \\
(36 \downarrow)\end{array}$ & $\begin{array}{c}25.3 \pm \\
0.42 \\
(55.2 \\
\downarrow) \\
\end{array}$ & $\begin{array}{c}23.5 \pm \\
0.26 \\
(69 \downarrow)\end{array}$ & $\begin{array}{c}27.1 \pm \\
0.19 \\
(70.4 \\
\downarrow) \\
\end{array}$ & $\begin{array}{c}77.5 \pm \\
0.11 \\
(8 \downarrow)\end{array}$ & $\begin{array}{c}74.7 \pm \\
0.09 \\
(10.7 \\
\downarrow) \\
\end{array}$ & $\begin{array}{c}71.2 \pm \\
0.08 \\
(12.1 \\
\downarrow) \\
\end{array}$ & $\begin{array}{c}72.9 \pm \\
0.11 \\
(13.3 \\
\downarrow) \\
\end{array}$ \\
\hline $\mathrm{CD} @ 5 \%$ & 0.35 & 0.34 & 0.32 & 0.26 & 0.26 & 0.35 & 0.32 & 0.52 & 0.24 & 0.16 & 0.45 & 0.36 \\
\hline
\end{tabular}


T0- Untreated Paddy straw (control)

T1- Paddy straw + FYM(3:1)

T2- Paddy straw + Fungal culture (5\%)

T3- Paddy straw + FYM(3:1) + Fungal culture $(5 \%)$

T4- Paddy straw + FYM(3:1) + Fungal culture $(5 \%)+$ Urea $(1 \%)$

T5- Paddy straw + FYM(3:1) + Fungal culture (5\%) + Urea (1\%) + Rock phosphate (1\%)

CD: Critical difference for triplicate data

\pm values indicate $\%$ Standard error for triplicate data

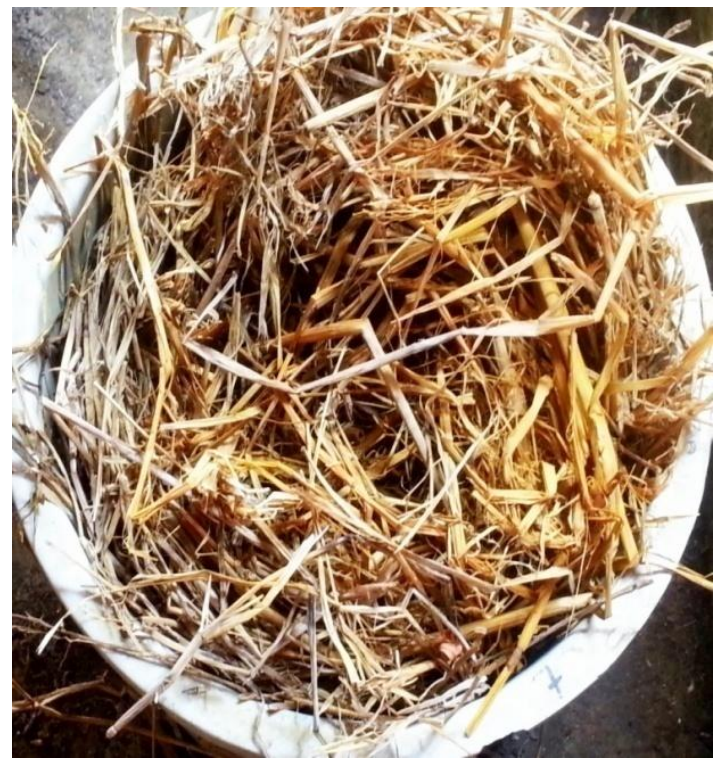

Control

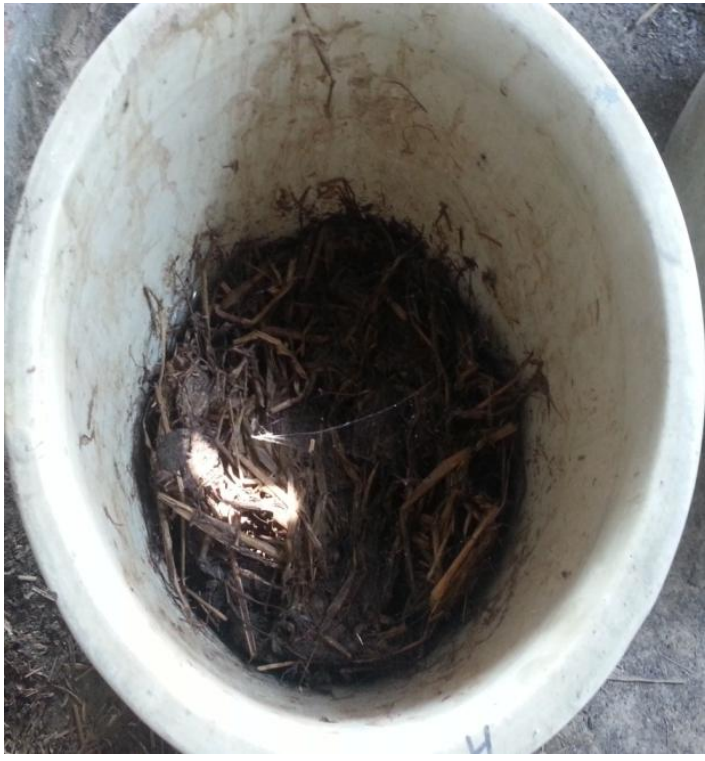

T5

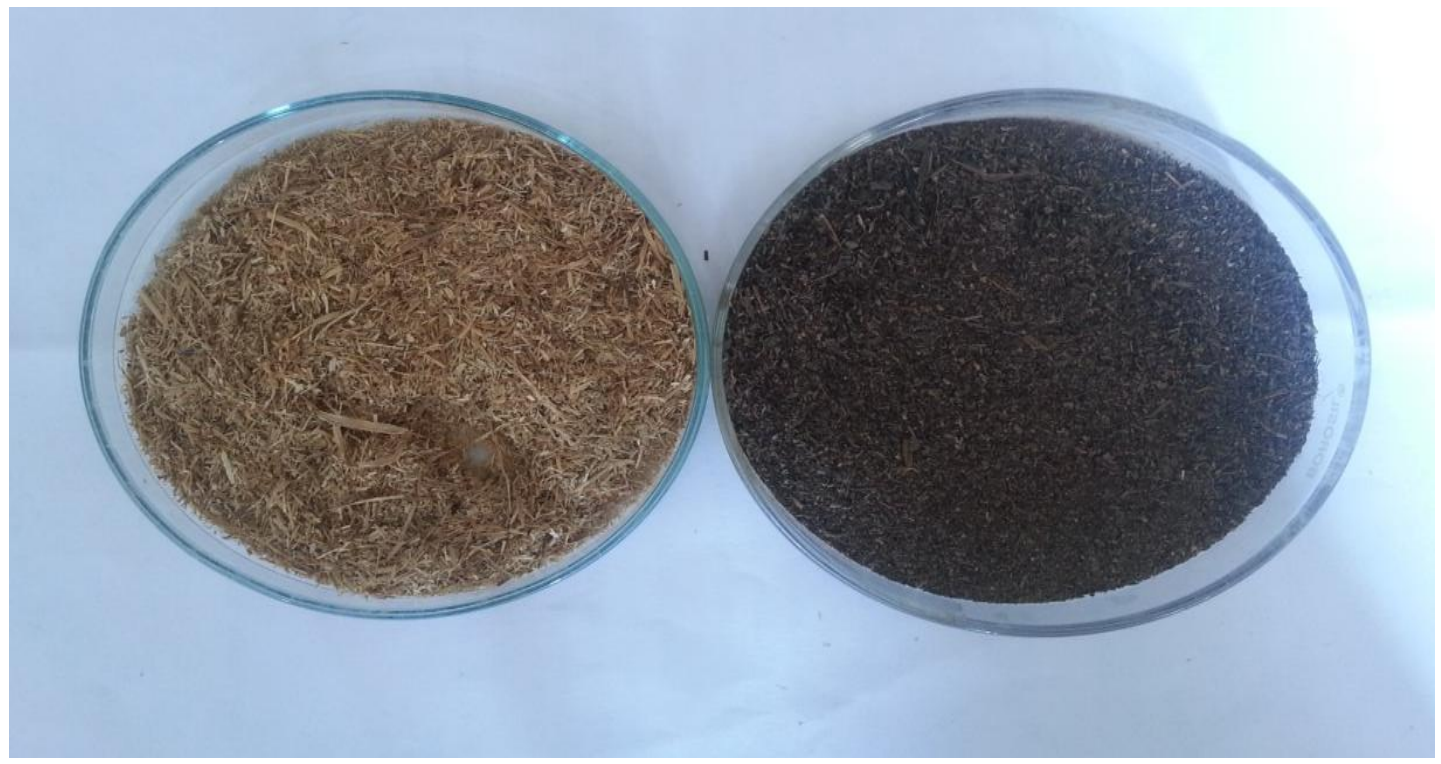

After grinding

Plate 1:- Composting of paddy straw by Treatment 5 (Paddy straw + Farm yard manure + Fungal culture + Urea + Rock phosphate) after 60 days. 


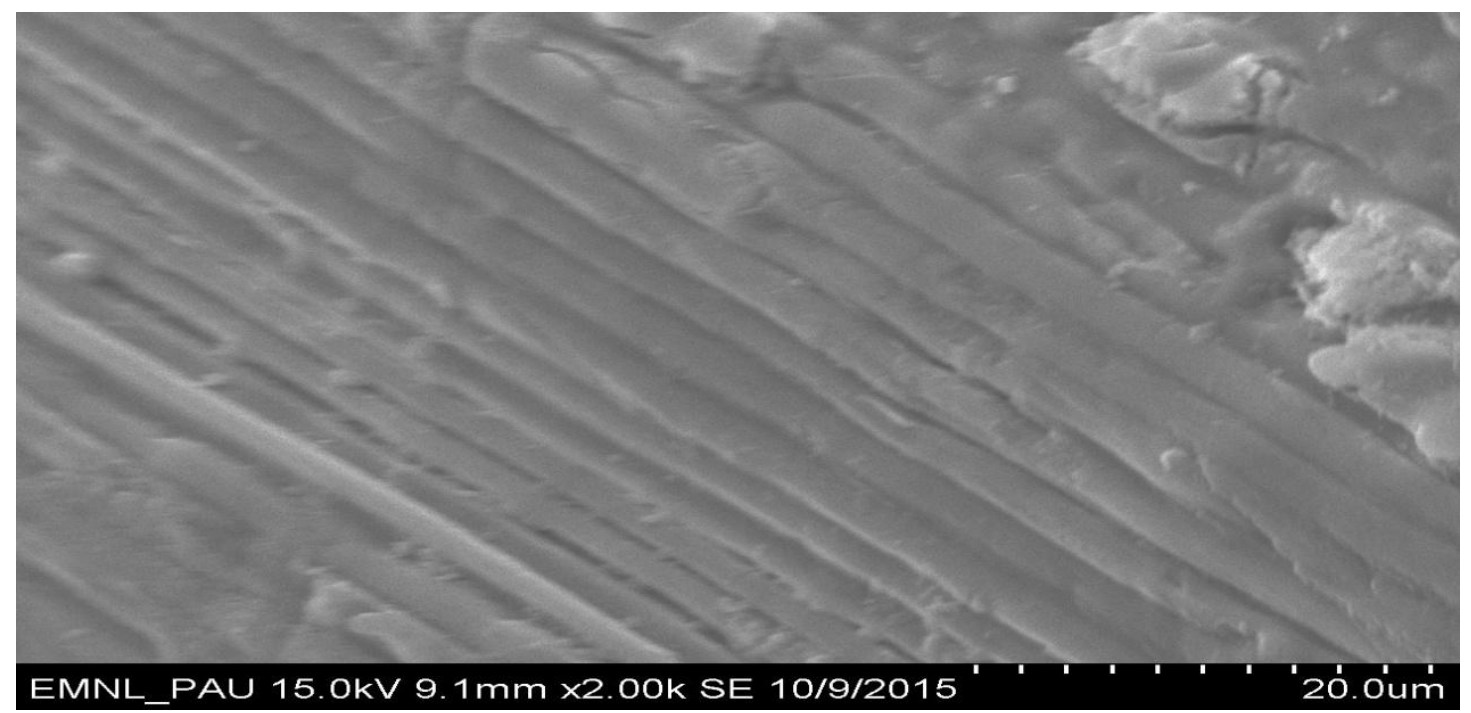

(a) Longitudinal section of paddy straw before treatment
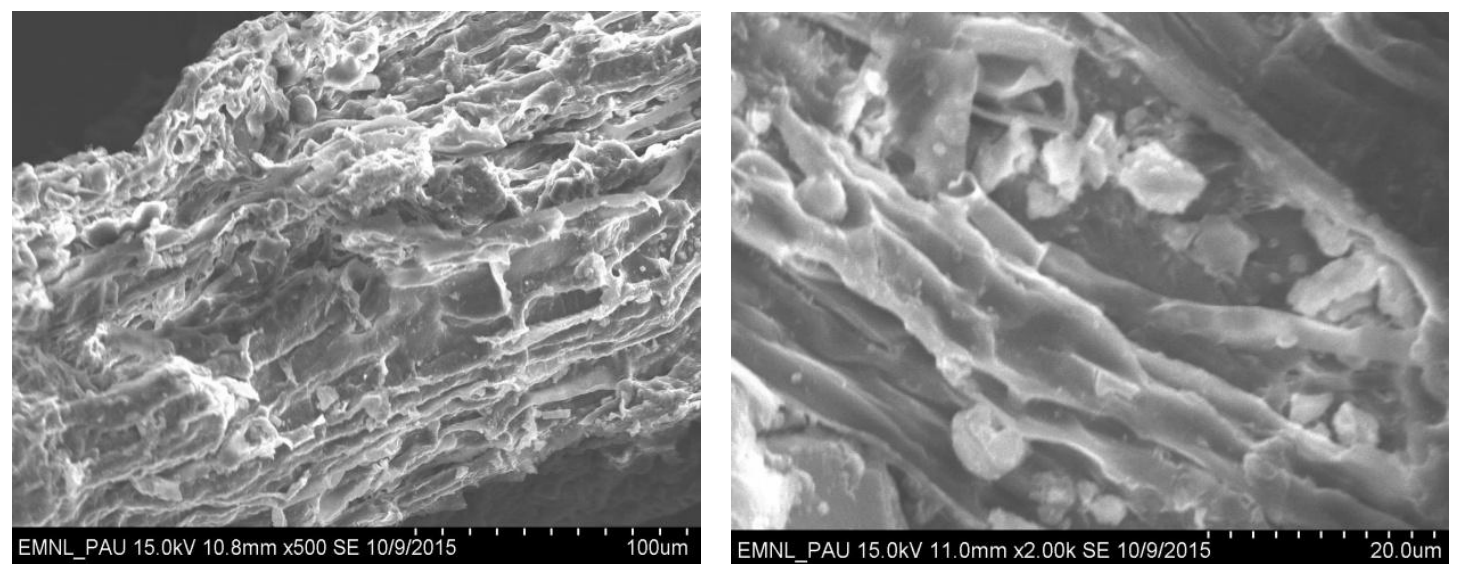

(b) Longitudinal section of paddy straw after treatment with T5 (Paddy straw + FYM + Fungal culture + Urea + Rock phosphate

Plate 2:- Scanning electron micrographs (SEM) of paddy straw before and after biological pretreatment, (a) longitudinal section of paddy straw before treatment, (b) longitudinal section of paddy straw after treatment with T5 (Paddy straw + FYM + Fungal culture + Urea + Rock phosphate.

\section{References:-}

1. Anonymous. (2015): Commodity profile for rice., pp: 2-9.

2. Anonymous. (2015): Management and utilization of paddy straw in Punjab. Department of Science, Technology \& Environment, Government of Punjab., Pp: 1-23.

3. Pandey, A.K., Gaind, S., Ali, A. and Nain, L. (2009): Effect of bioaugmentation and nitrogen supplementation on composting of paddy straw. Biodegrad., 20: 293-306.

4. Gadde, B., Bonnet, S., Menke, C. and Garivait, S. (2009): Air pollutant emissions from rice straw open field burning in india, Thailand and Philippines. Environ. poll., 157: 1554-58.

5. Jalk. D., Nerud, R. and Siroka, P. (1998): The effectiveness of biological treatment of wheat straw by white rot Fungi. Folia. Microbiol., 43: 687-89.

6. Eriksson, K-EL., Blanchette, R.A. and Ander, P. (1990): Microbial and enzymatic degradation of wood and wood components. Springer Berlin Heidelberg, New York.

7. Okano, K., Kitagawa, M., Sasaki, Y. and Watanabe, T. (2005): Conversion of Japanese red cedar(Cryptomeria japonica) into a feed for ruminants by white-rot basidiomycetes. Anim. Feed. Sci. Technol., 120: 235-43. 
8. Taniguchi, M., Suzuki, H., Watanabe, D., Sakai, K., Hoshino, K. and Tanaka, T. (2005): Evaluation of pretreatment with pleurotus ostreatus for enzymatic hydrolysis of rice straw. J. Biosci. Bioeng., 100: 637-43.

9. Coughlan, M. (1985): The properties of fungal and bacterial cellulases with comment on their activities and application. Biotechnol. Genet. Engr. Rev., 3: 39-109.

10. Rodrigues, M. A. M., Pinto, P., Bezerra, R. M. F., Dias, A. A. and Guedes, C. V. M. (2008): Effect of enzyme extracts isolated from white rot fungi and in vitro digestibility of wheat straw. Anim Feed Sci Technol 141: 326-38.

11. Panagiotou, G., Olavarria, R. and Olsson, L. (2007): Penicillium brasilianum as an enzyme factory; the essential role of feruloyl esterases for the hydrolysis of the plant cell wall. J. Biotechnol., 130: 219-28.

12. Fazaeli, H. (2007): Nutritive value index of treated wheat straw with Pleurotus fungi. Biotechnol. Ani .Husband., 23: 169-80.

13. Mylavarapu, R. S. and Zinati, G. M. (2009): Improvement of soil properties using compost of optimum parsley production in sandy soils. Scientia. Horticult., 120: 426-30.

14. Zibiliske, L .M. (1998): Composting of organic wastes. Pp 402. Lewis publishers, Boca Raton, Florida.

15. Sun, Y. and Cheng. J, (2002): Hydrolysis of lignocellulosic materials for ethanol production. Rev. Bioresour. Technol., 83: 1-11.

16. Phutela, U. G., Sahni, N. and Sooch, S.S. (2011): Fungal degradation of paddy straw for enhancing biogas production. Ind. J. Sci. Technol., 4: 660-65.

17. Scott, G. M., Akhtar, M., Lentz, M. J. and Kirk, T. K. (1998): New technology for papermaking commercializing biopulping. J. Tappi., 81: 220-25.

18. Dhaliwal, R. P. S., Garcha, H. S and Khanna, P. K. (1991): Regulation of lignocellulytic enzyme system in P. ostreatus. Ind. J. Microbiol., 31: 181-84.

19. Sandhu, D. K. and Kalra, M. K. (1982): Production of cellulase, xylanase and pectinase by Trichoderma longibrachiatum on different substrates. Trans. British. Mycol. Soc. 79: 409-13.

20. AOAC. Association of Official Analytical Chemists, Official Methods of Analysis, 17th Edition, Maryland, USA; 2000.

21. Chmelova,D., Ondrejovic, M., Ondas,V., Sturdik, E. (2011): Influence of cultivation conditions on production of lignocellulolytic enzymes by Ceriporiopsis subvermispora. Biol., 66: 748-54.

22. Mishra, B. K., Pandey, A. K. and Lata. (2007): Lignocellulolytic Enzyme Production from Submerged Fermentation of Paddy Straw. Ind. J. Microbiol., 47: 176-79.

23. Prashanthi, S. G., Gentela, J., Koti, S., Haragopal, V., Venkateshwar, S. and Venkateswar, L. R. (2015): Extracellular Lignocellulolytic Enzymes by Phanerochaete chrysosporium (MTCC 787) Under SolidState Fermentation of Agro Wastes. Int. J. Curr. Microbiol. App. Sci., 10:700-10.

24. Goyal, S., Dhull, S. K. and Kapoor, K. K. (2005): Chemical and biological changes during composting of different organic wastes and assessment of compost maturity. Bioresour. Technol., 96: 1584-91.

25. Beary, T. P., Boopathy, R. and Templet. P. (2002): Accelerated decomposition of sugarcane crop residue using a fungalbacterial consortium. Int. Biodet. Biodeg., 50: 41-6.

26. Yu, M., Zeng, G., Chen, Y., Yu, H., Huang, D. and Tang, L. (2009): Influence of Phanerochaete chrysosporium on microbial communities and lignocelluloses degradation during solid state fermentation of rice straw. Process. Biochem., 44: 17-22. 